\title{
Laser Acceleration of Electrons: Zero to $c$ in Less than Ten Microns
}

\author{
Donald Umstadter \\ Center for Ultrafast Optical Science ${ }^{1}$ \\ University of Michigan, \\ Ann Arbor, MI 48109
}

\begin{abstract}
It has recently been shown that by simply focusing a high-intensity laser into a gas jet, a well-defined and low-divergence beam of relativistic electrons is produced, which is accelerated by a laser wakefield. Above a certain power threshold, the laser is observed to be relativistically self-guided, creating its own light pipe. This effectively increases the laser propagation distance (beyond the fundamental diffraction limit), decreases the electron beam divergence, and increases the electron energy.
\end{abstract}

\section{INTRODUCTION}

Due to recent advances in laser technology [1], it is now possible to generate-in the interactions of high-intensity and ultrashort-duration laser pulses with matter - the highest electromagnetic and electrostatic fields ever produced in the laboratory [2]. This has come to be known as high-field science. In terms of basic research, these interactions permit for the first time the study of optics in relativistic plasmas. Technological applications include advanced fusion energy, $\mathrm{x}$-ray lasers, and table-top ultrahigh-gradient electron accelerators. We discuss several aspects that are especially pertinent to the latter.

When an intense laser enters a region of gaseous-density atoms, the atomic electrons feel the enormous laser electromagnetic field, and begin to oscillate at the laser frequency $(2 \pi c / \lambda=c k)$. The oscillations can become so large that the electrons become stripped from the atoms, or ionized. At high laser intensity $(I)$, the free electrons begin to move at close to the speed of light $(c)$, and thus their mass $m_{e}$ changes significantly compared to their rest mass.

1) This work is sponsored by Department of Energy/Lawrence Livermore National Laboratory subcontract B307953 and the National Science Foundation Science and Technology Center contract PHY 8920108. 
This large electron oscillation energy corresponds to gigabar laser pressure, displacing the electrons from regions of high laser intensity. Due to their much greater inertia, the ions remain stationary, providing an electrostatic restoring force. These effects cause the plasma electrons to oscillate at the plasma frequency $\left(\omega_{p}\right)$ after the laser pulse passes by them, creating alternating regions of net positive and negative charge, where $\omega_{p}=\sqrt{4 \pi e^{2} n_{e} / \gamma m_{e}}, n_{e}$ is the electron density, $e$ is the electron charge and $\gamma$ is the relativistic factor associated with the electron motion transverse to the laser propagation. $\gamma$ depends on the normalized vector potential, $a_{o}$, by $\gamma=\sqrt{1+a_{o}^{2}}$, where $a_{o}=\gamma v_{o s} / c=e E / m_{o} \omega c=8.5 \times 10^{-10} \lambda[\mu \mathrm{m}] I^{1 / 2}\left[\mathrm{~W} / \mathrm{cm}^{2}\right]$. The resulting electrostatic wakefield plasma wave propagates at a phase velocity nearly equal to the speed of light and thus can continuously accelerate hot electrons [3]. Up to now, most experiments have been done in the self-modulated laser wakefield regime [4-6], where the laser pulse duration is much longer than the plasma period, $\tau \gg>\tau_{p}=2 \pi / \omega_{p}$. In this regime, the forward Raman scattering instability can grow; where an electromagnetic wave $\left(\omega_{o}, \boldsymbol{k}_{o}\right)$ decays into a plasma wave $\left(\omega_{p}, \boldsymbol{k}_{p}\right)$ and electromagnetic side-bands $\left(\omega_{o} \pm \omega_{p}, \boldsymbol{k}_{\boldsymbol{o}} \pm \boldsymbol{k}_{\boldsymbol{p}}\right)$.

\section{RECENT RESULTS}

A small number of relativistic hot electrons were observed in inertialconfinement-fusion experiments with long-pulse duration large-building size lasers and solid-density targets. However, it was shown only recently that electrons can be accelerated by a plasma wave driven by intense ultrashortduration table-top laser pulses ( $\mathrm{I} \sim 4 \times 10^{18} \mathrm{~W} / \mathrm{cm}^{2}, \lambda=1 \mu \mathrm{m}, \tau \sim 0.5 \mathrm{ps}$ ) and gaseous-density targets $[7,8]$. Under similar conditions, electrons were even observed to have an energies up to $44 \mathrm{MeV}$, with an energy spread of $100 \%$ [9]. It was then shown that the accelerated electron beam appeared to be naturally-collimated with a low-divergence angle (less than ten degrees), and had over 1-nC of charge per bunch [10]. Moreover, as shown in Fig. 1, acceleration occured in this experiment [10] only when the laser power exceeded a certain critical value, $P_{c}$, the threshold for relativistic self-focusing. Since then, two independent research groups have simultaneously reported direct measurements of the plasma wave amplitude with a Thomson-scattering probe pulse $[11,12]$. The field gradient was reported [12] to exceed that of a radio-frequency (RF) linac by four orders of magnitude $(E \geq 200 \mathrm{GV} / \mathrm{m})$. This acceleration gradient corresponds to an energy gain of $1 \mathrm{MeV}$ in a distance of only 10 microns. The plasma wave was observed to exist for a duration of $1.5 \mathrm{ps}$ or 100 plasma oscillations [12]. It was calculated that it damps only because all of the wave energy was converted to the accelerated electrons. Except for the large energy spread and low average power, these parameters compare favorably with medical linacs. In fact, the much smaller source size of a laser wakefield accelerator compared with that of a conventional linac, 10 


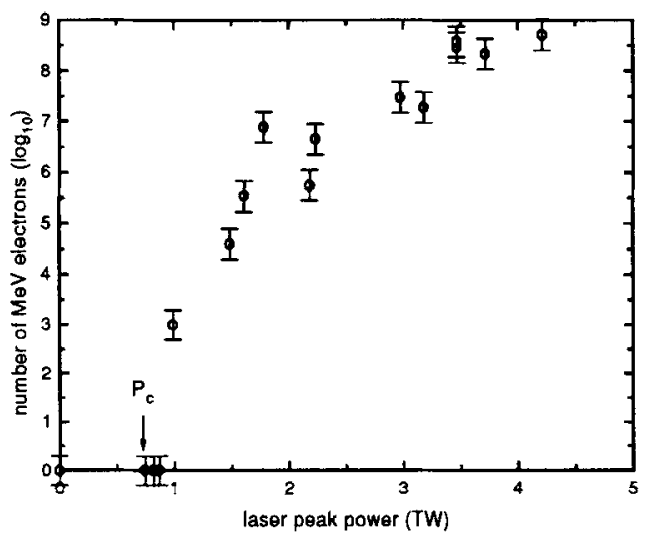

FIGURE 1. The number of relativistic electrons accelerated as a function of incident laser power focused in a gas of helium at atmospheric density.

microns compared with greater than 100 microns, may permit much greater spatial resolution for medical imaging.

This enormous field gradient would be of limited use if the length over which it could be used to accelerate electrons were just the natural diffraction length of the highly focused laser beam, which is much less than a millimeter. Fortunately, it was recently demonstrated that electrons can be accelerated beyond this distance [13]. At high laser power, the index of refraction in a plasma varies with the radius. This is both because the laser intensity varies with radius and the plasma frequency depends on the relativistic mass factor $\gamma$. Above the above-mentioned critical laser power $P_{c}$, the plasma should act like a positive lens and focus the laser beam, a process called relativistic selffocusing. This is similar to propagating a low power beam over an optical fiber optic cable, except in this case the intense laser makes its own fiber optic. The relativistically self-guided channel was found to increase the laser propagation distance by a factor of four (limited thus far only by the length of gas), decrease the electron beam divergence by a factor of two (as shown in Fig. 2), and increase the electron energy.

\section{FUTURE DIRECTIONS}

In the above experiments, the plasma itself acted as the cathode (the source of the electrons). Since the electrons were picked up by the wave and accelerated with random phases, their energy spread was large. This may be acceptable for medical radiological sources, where broadband bremstrahlung $\mathrm{x}$-ray radiation is created anyway, by focusing the electron beam onto a metal target. However, in order to create monoenergetic femtosecond duration electron bunches, a new concept for laser injection of electrons was developed (as 


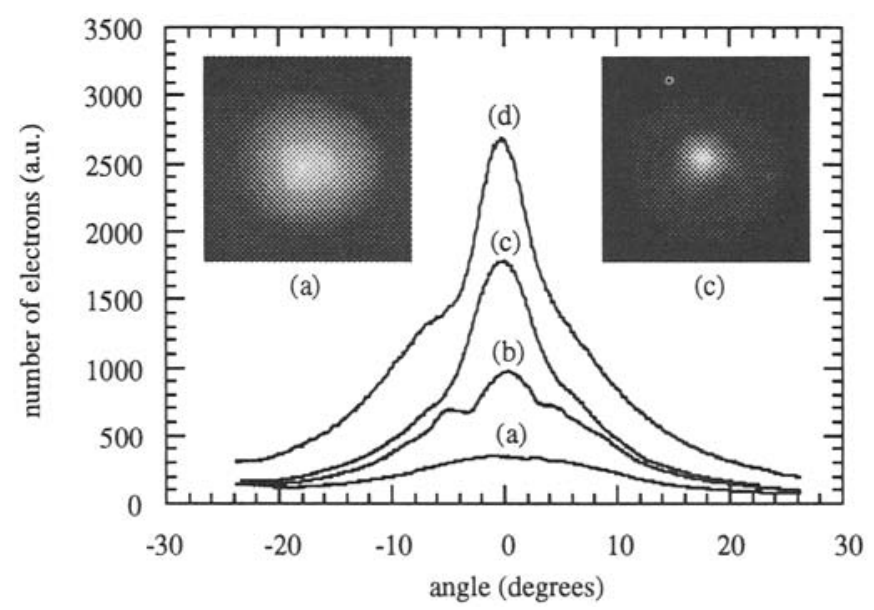

FIGURE 2. Electron beam divergence as a function of laser power. The various curves represent laser powers of $P / P_{c}=$ (a) 3.4, (b) 5.0, (c) 6.0, and (d) 7.5. The two insert figures show the complete beam images for curves (a) and (c).

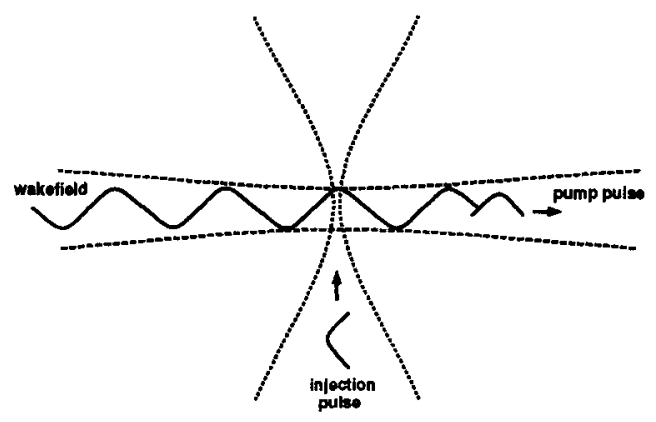

FIGURE 3. Schematic of the crossed-laser-plasma wave accelerator concept.

shown in Fig. 3), again using the plasma itself as the cathode but with laser triggering [14]. Either transverse (as shown) or collinear laser beams can be used. This is similar to giving several surfers identical pushes so that they all catch the same ocean wave in phase with one another. It was shown by use of a numerical code that with this method and by operating in the resonant wakefield regime $\left(\tau \sim \tau_{p}\right)$, it should be possible to produce femtosecond electron bunches with energy spread at the percent level.

As an injector stage for linear electron colliders for nuclear and highenergy physics, this pulse length reduction may have several interesting consequences. In the case of an electron-electron linear collider, it would have a higher limit on attainable luminosity by permitting a shorter $\beta$ (in effect, the Rayleigh parameter of the magnetic optics) at the final focus/intersection point and it would also reduce beam-beam effects by reducing the time during 
which the beams overlap. There would also be a reduction in beam-beam Bremsstrahlung ("beamstrahlung") due to quantum mechanical effects. Such ultrashort-duration electron bunches may also become the basis for a new generation of table-top radiation sources. They may increase both the coherence and gain of synchrotrons, free-electron lasers, or Compton-scattering sources. Additionally, the ultrashort light-pulses that they may provide might be used to as "strobes" to probe temporal dynamics on the natural timescale of important ultrafast chemical, biological and physical processes.

Another factor limiting the applicability of these laser accelerators, low average power, will be overcome as laser technology improves. With diodepumping reaching maturity, it is expected that within the next couple of years the required laser peak intensities will be reached with 100 watt laser systems. This will be an improvement by a factor of $10^{5}$ over the average power of the systems used in these proof-of-principle experiments, and close that of conventional RF technology.

\section{REFERENCES}

1. P. Maine, D. Strickland, P. Bado, M. Pessot and G. Mourou, IEEE J. Quantum Electron. QE-24, 398 (1988).

2. G. Mourou and D. Umstadter, Phys. Fluids B 4, 2315 (1992).

3. T. Tajima and J. M. Dawson, Phys. Rev. Lett. 43, 267 (1979).

4. N. E. Andreev, L. M. Gorbunov, V. I. Kirsanov, A. Pogosova and R. R. Ramazashvili, Pis'ma Zh. Eksp. Teor. Fiz., 55551 (1992) [JETP Lett., 55, 571 (1992)].

5. T. M. Antonsen, Jr., and P. Mora, Phys. Rev. Lett., 69, 2204 (1992).

6. P. Sprangle, E. Esarey, J. Krall, and G. Joyce, Phys. Rev. Lett., 69, 2200 (1992).

7. C. A. Coverdale, C. B. Darrow, C. D. Decker, W. B. Mori, K. -C. Tzeng, K. A. Marsh, C. E. Clayton, and C. Joshi, Phys. Rev. Lett. 74, 4659, (1995).

8. K. Nakajima, D. Fisher, T. Kawakubo, H. Nakanishi, A. Ogata, Y. Kato, Y. Kitagawa, R. Kodama, K. Mima, H. Shiraga, K. Suzuki, K. Yamakawa, T. Zhang, Y. Sakawa, T. Shoji, N. Yugami, M. Downer and T. Tajima, Phys. Rev. Lett. 74, 4428, (1995).

9. A. Modena, Z. Najmudin, A. E. Dangor, C. E. Clayton, K. A. Marsh, C. Joshi, V. Malka, C. B. Darrow, C. Danson, D. Neely and F. N. Walsh, Letts. Nature 377, 606, (1995).

10. D. Umstadter, S.-Y. Chen, A. Maksimchuk, G. Mourou, and R. Wagner, "Nonlinear Optics in Relativistic Plasmas and Laser Wakefield Acceleration of Electrons," Science 273, 472 (1996).

11. A. Ting, K. Krushelnick, C. I. Moore, H. R. Burris, E. Esarey, J. Krall, and P. Sprangle, "Temporal Evolution of Self-Modulated Laser Wakefields Measured by Coherent Thomson Scattering," Phys. Rev. Lett. 77, 5377 (1996). 
12. S. P. Le Blanc, M. C. Downer, R. Wagner, S.-Y. Chen, A. Maksimchuk, G. Mourou and D. Umstadter, "Temporal Characterization of a Self-Modulated Laser Wakefield," Phys. Rev. Lett. 77, 5381 (1996).

13. R. Wagner, S.-Y. Chen, A. Maksimchuk and D. Umstadter, "Relativistically Self-Guided Laser Wakefield Acceleration," Phys. Rev. Lett. (accepted for publication, 1997).

14. D. Umstadter, J. K. Kim, and E. Dodd, "Laser Injection of Ultrashort Electron Pulses into Wakefield Plasma Waves," Phys. Rev. Lett. 76, 2073 (1996). 